\title{
The Advanced URANUS Navier-Stokes Code for the Simulation of Nonequilibrium Re-entry Flows
}

\author{
By Markus FERTIG ${ }^{1)}$ and Georg HeRdRICH${ }^{1)}$
}

1) Institut für Raumfahrtsysteme, Universität Stuttgart, Germany

(Received April 25th, 2008)

\begin{abstract}
In order to predict the thermal and mechanical loads during re-entry, the URANUS (Upwind Relaxation Algorithm for Nonequilibrium Flows of the University of Stuttgart) has been being developed at the Institute of Space Systems (IRS) of the Universität Stuttgart. For the accurate determination of the thermochemical conditions, advanced thermochemical relaxation models for the gas-phase as well as sophisticated gas-surface interaction models have been developed. The Navier-Stokes equations for the 11-component air flow, which consists of $\mathrm{N}_{2}, \mathrm{O}_{2}, \mathrm{NO}, \mathrm{N}, \mathrm{O}, \mathrm{N}_{2}^{+}$, $\mathrm{O}_{2}^{+}, \mathrm{NO}^{+}, \mathrm{O}^{+}, \mathrm{N}^{+}$and $\mathrm{e}^{-}$, have been derived by the Chapman-Enskog method from the Boltzmann-Equation. The linearized system of equations is solved fully coupled and fully implicitly, employing Newton's method. In the paper, the modelling is described in some detail and selected simulation results are presented.
\end{abstract}

Key Words: Nonequilibrium, Re-entry, CFD, Boltzmann, Chapman-Cowling

\section{Nomenclature}

$$
\begin{aligned}
& a_{i m} \text { :Sonine coefficient }\left(\mathrm{m} \mathrm{K}^{-1}\right) \\
& b \text { :collision parameter }(\mathrm{m}) \\
& b_{i m} \text { :Sonine coefficient (s) } \\
& \vec{c} \text { :molecular velocity }\left(\mathrm{m} \mathrm{s}^{-1}\right) \\
& c_{p} \text { :specific heat capacity at constant pressure } \\
& \left(\mathrm{J} \mathrm{kg}^{-1} \mathrm{~K}^{-1}\right) \\
& c_{v} \text { :specific heat capacity at constant volume } \\
& \left(\mathrm{J} \mathrm{kg}^{-1} \mathrm{~K}^{-1}\right) \\
& c_{i m}^{(h, k)}: \text { Sonine coefficient }\left(\mathrm{m}^{3}\right) \\
& D \text { :multi component diffusion coefficient } \\
& \left(\mathrm{m}^{2} \mathrm{~s}^{-1}\right) \\
& D^{T} \text { :thermal diffusion coefficient }\left(\mathrm{kg} \mathrm{m}^{-1} \mathrm{~s}^{-1}\right) \\
& \mathscr{D} \text { :binary diffusion coefficient }\left(\mathrm{m}^{2} \mathrm{~s}^{-1}\right) \\
& \overline{\bar{E}} \text { :unit matrix }(-) \\
& \overrightarrow{\mathcal{F}} \text { :inviscid flux vectors } \\
& \overrightarrow{\mathcal{F}}_{v} \text { :viscous flux vectors } \\
& \eta \text { :coefficient of viscosity }\left(\mathrm{kg} \mathrm{m}^{-1} \mathrm{~s}^{-1}\right) \\
& \varepsilon_{e l}(l) \text { :electronic energy in quantum state } l(\mathrm{~J}) \\
& \varepsilon_{\text {int }} \text { :energy of an internal quantum state }(\mathrm{J}) \\
& \varepsilon_{\text {rot }}(J) \text { :rotational energy in quantum state } J(\mathrm{~J}) \\
& \varepsilon_{v i b}(v) \text { :vibrational energy in quantum state } v(\mathrm{~J}) \\
& e \text { :mass specific energy }\left(\mathrm{J} \mathrm{kg}^{-1}\right) \\
& F \text { :external force }(\mathrm{N}) \\
& f \text { :velocity distribution function }\left(\mathrm{s}^{3} \mathrm{~m}^{-6}\right) \\
& f_{\text {int }} \text { :distribution function for an internal degree of } \\
& \text { freedom (int } \in\{\text { rot }, \text { vib }, e l\})(-) \\
& g \text { :relative velocity }\left(\mathrm{ms}^{-1}\right) \\
& \Delta H_{f}^{0} \text { :standard enthalpy of formation }\left(\mathrm{kJ} \text { mole }{ }^{-1}\right) \\
& J \text { :rotational quantum number (-) }
\end{aligned}
$$

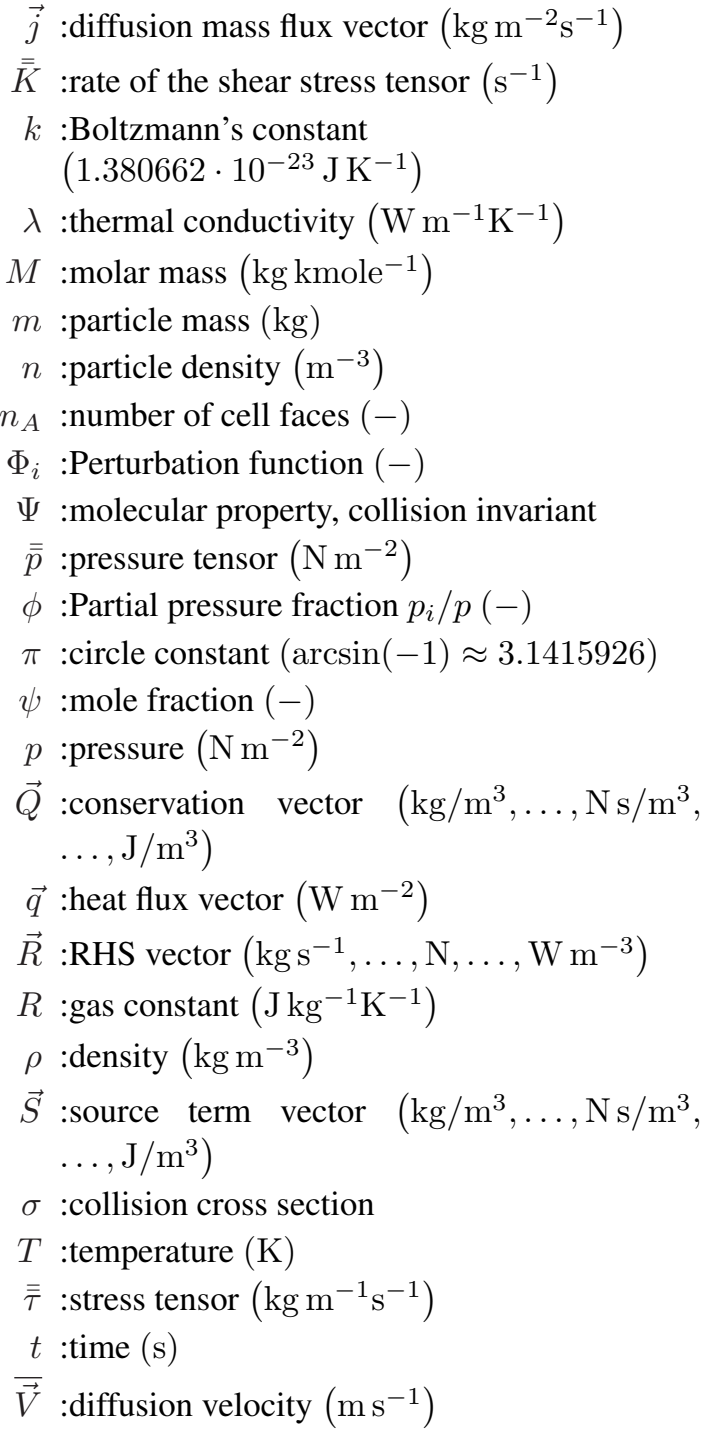




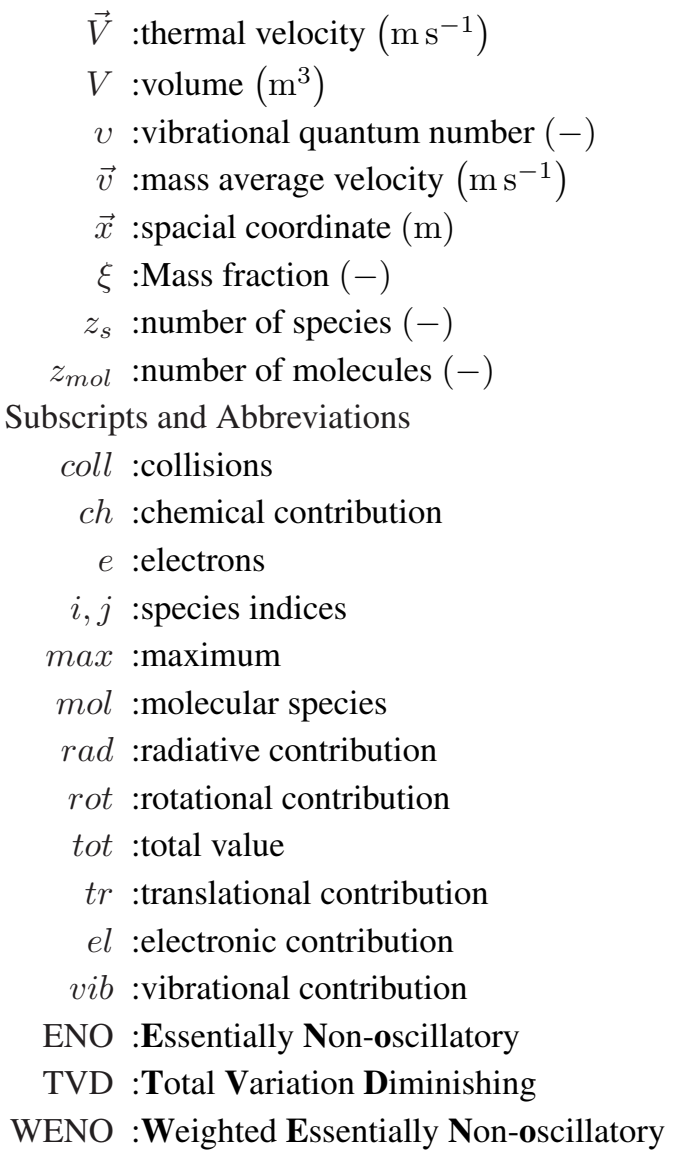

\section{Introduction}

During re-entry of a space vehicle into the atmosphere, high thermal and mechanical loads arise. Typical re-entry velocities from low earth orbit (LEO) are about $8 \mathrm{~km} / \mathrm{s}$ leading to a specific gas enthalpy of $32 \mathrm{MJ} / \mathrm{kg}$. Due to the high velocity, a compression shock forms in front of the vehicle. As a consequence, temperature and pressure increase by factors of approximately 250 and 1000, respectively. With rising atmospheric density the speed of the vehicle decreases. For ballistic vehicles the thermal loads reach a maximum at about $60 \mathrm{~km}$ of altitude, where the velocity is about $6 \mathrm{~km} / \mathrm{s}$. Under these conditions pressure increases to about $20 \mathrm{kPa}$ and translational temperature increases to about $20000 \mathrm{~K}$ across the normal shock in front of the vehicle. Downstream of the shock, relaxation of internal degrees of freedom like rotation and vibration as well as chemistry arises. Roughly $2 / 3$ of the energy of the high-enthalpy gas flow is converted into chemical enthalpy by chemical reactions, leading to the temperature being reduced to about $6000 \mathrm{~K}$. Within the boundary layer the temperature is reduced to about $2000 \mathrm{~K}$ at the surface. Under peak heating conditions, the reaction velocity in the boundary layer is not high enough to obtain chemical equilibrium. Hence, the gas flow reaching the surface of the vehicle may be mainly composed of highly reactive atoms. Recombination of atoms at the thermal protection system (TPS) may release the energy stored in the chemical composition of the gas, hereby increasing the thermal loads of the surface. Restricting the investigation to non-ablative
TPS materials, convective heat transfer and catalysed reactions at the surface dominate the heat flux.

The re-entry from interplanetary missions leads to even more challenging problems. Since the entry speed is about $12 \mathrm{~km} / \mathrm{s}$, a specific gas enthalpy of $72 \mathrm{MJ} / \mathrm{kg}$ arises. Due to the higher speed, the compression ratio across the shock becomes as high as 2000 and the post shock temperatures can reach $100000 \mathrm{~K}$. The post shock relaxation leads to a decrease of temperature down to approximately $12000 \mathrm{~K}$. Since an interplanetary re-entry vehicle is usually smaller than re-entry vehicles returning from LEO, the stagnation point's heat flux may exceed $10 \mathrm{MW} / \mathrm{m}^{2}$. In comparison to an entry from LEO, ionisation reactions and radiation effects become an important issue. While the convective heat flux depends primarily on the enthalpy and density of the atmosphere as well as on the geometry of the vehicle, the radiative heat flux is additionally much more dependent on the composition behind the shock. The situation is further complicated if the thermo-chemical state of the gas is not in equilibrium, since in this case simplifications of the distribution of internal energy are questionable.

In order to predict the thermal and mechanical loads during re-entry, URANUS (Upwind Relaxation Algorithm for Nonequilibrium Flows of the University on Stuttgart) has been being developed at the Institute of Space Systems (IRS) of the Universität Stuttgart. For the accurate determination of the thermochemical conditions, advanced thermochemical relaxation models for the gas-phase ${ }^{1,2,3,4,5)}$ as well as sophisticated gas-surface interaction models have been being developed. $6,7,8,9,10,11,12$ )

\section{Transport Equations}

From the microscopic point of view, a particle without internal degrees of freedom can be characterised by its mass and velocity. Combining all particles with equal mass within a volume element, a phase space distribution function

$$
f_{i}\left(\vec{x}, \vec{c}_{i}, t\right)
$$

can be defined. From Eq. (1) mean macroscopic gas properties can be found by evaluation of

$$
\begin{aligned}
\rho & =\sum_{i} m_{i} \int f_{i} d \vec{c}_{i} \quad \text { for the density, } \\
\vec{v} & =\frac{1}{\rho} \sum_{i} m_{i} \int \vec{c}_{i} f_{i} d \vec{c}_{i} \quad \text { for the velocity, } \\
T & =\frac{m}{3 n k} \sum_{i} \int V_{i}^{2} f_{i} d \vec{V}_{i} \quad \text { for the temperature and } \\
\overline{\bar{p}} & =\sum_{i} m_{i} \int \vec{V}_{i} \vec{V}_{i}^{T} f_{i} d \vec{V}_{i} \text { for the pressure }
\end{aligned}
$$

where the thermal velocity $\vec{V}_{i}$ is defined by

$$
\vec{V}_{i}=\vec{c}_{i}-\vec{v},
$$

and $n_{i}$ denotes the species number density, which is given by $n_{i}=\int f_{i} d \vec{c}_{i}$. A general equation describing the change 
of the distribution function is the Boltzmann equation

$$
\frac{\partial f_{i}}{\partial t}+\vec{c}_{i} \cdot \nabla_{x} f_{i}+\frac{\vec{F}_{i}}{m_{i}} \cdot \nabla_{c} f_{i}=\left(\frac{\delta f_{i}}{\delta t}\right)_{\text {Coll }},
$$

which describes the change of the distribution function in time and phase space as a result of external forces and particle collisions, depending on local velocity. The term on the right-hand side of Eq. (7) represents the Boltzmann collision integral ${ }^{13}$ )

$$
\left(\frac{\delta f_{i}}{\delta t}\right)_{C o l l}=2 \pi \sum_{j=1}^{z_{s}} \iint\left(f_{i}^{\prime} f_{j}^{\prime}-f_{i} f_{j}\right) g_{i j} b d b d \vec{c}_{j},
$$

which reflects the rate of change of $f_{i}$ with respect to time due to collisions. The prime refers to the value of a quantity after a collision and unprimed symbols denote the values before the collision. From the mathematical point of view, the Boltzmann equation is a very complicated integrodifferential equation which can be used to determine the velocity distribution function. Up to now, a general solution of the Boltzmann equation to describe macroscopic problems is not possible. Therefore, several simplifications are necessary to compute solutions for the Boltzmann equation.

Both Chapman ${ }^{14)}$ and Enskog ${ }^{15}$ ) developed independently of each other a method for approximately solving the Boltzmann Equation by expressing it as an infinite series. The first term of this series, which represents the equilibrium solution, can be found quite easily by setting both sides of the Boltzmann Equation equal zero. This results in the well-known Maxwell distribution function

$$
f_{i}^{[0]}=n_{i}\left(\frac{m_{i}}{2 \pi k T_{i}}\right)^{\frac{3}{2}} e^{-\frac{m_{i} V_{i}^{2}}{2 k T_{i}}} .
$$

The following elements of the Enskog-Series result in subsequent approximation of the velocity distribution function in nonequilibrium. Limiting the series to two elements, one finds

$$
f_{i} \approx f_{i}^{[1]}=f_{i}^{[0]}\left[1+\Phi_{i}\right],
$$

where the perturbation function $\Phi_{i}$ has to be determined by solving the collision integral (8).

Expressing the pertubation function by Sonine polynomials as explained by Hirschfleder et al. ${ }^{13)}$, one obtains

$$
\begin{aligned}
f_{i} \approx f_{i}^{[0]} & \left(1-a_{i 0}(\alpha) \vec{W}_{i}^{T} \cdot \frac{\partial \ln T_{i}}{\partial \vec{x}}\right. \\
& -a_{i 1}(\alpha)\left(\frac{5}{2}-W_{i}^{2}\right) \vec{W}_{i}^{T} \cdot \frac{\partial \ln T_{i}}{\partial \vec{x}} \\
- & b_{i 0}(\alpha)\left(\vec{W}_{i} \cdot \vec{W}_{i}^{T}-\frac{1}{3} W_{i}^{2} \overline{\bar{E}}\right): \frac{\partial}{\partial \vec{x}} \vec{v}^{T} \\
& \left.+n \sum_{j}\left(c_{i 0}^{(j, i)}(\alpha) \vec{W}_{i}^{T} \cdot \vec{d}_{j}\right)\right)
\end{aligned}
$$

for the second approximation to the velocity distribution function, where

$$
\vec{W}_{i}=\vec{V}_{i} \sqrt{\frac{m_{i}}{2 k T_{i}}}
$$

is the reduced velocity. The vector

$$
\overrightarrow{d_{j}}=\frac{\partial \phi_{j}}{\partial \vec{x}}+\left(\phi_{j}-\xi_{j}\right) \frac{\partial \ln p}{\partial \vec{x}}-\frac{\xi_{j}}{p}\left[\frac{\rho}{m_{j}} \vec{F}_{j}-\sum_{k} n_{k} \vec{F}_{k}\right]
$$

is the gradient vector for diffusion and contains contributions of mass diffusion, pressure diffusion as well as of external forces $\vec{F}_{j}$. Note that the determination of the diffusion gradient vector results in the partial pressure fraction $\phi_{i}=p_{i} / p$ as the gradient vector for mass diffusion in a multi-temperature environment.

The Sonine coefficients result from an infinite, fast converging series. Hence, these coefficients depend on the number of expansion terms $\alpha$ of the Sonine expansions. Note that the coefficients $a_{i 0}$ and $a_{i 1}$ become zero for $\alpha<2$. In literature, two different notations for the order of approximation can be found. While Chapman and Cowling $\left.{ }^{14}\right)$ denote $a_{i 1}(\alpha=2)$ the first approximation, Hirschfelder et al. ${ }^{13)}$ use the value of $\alpha$, which is preferred here as well. Usually, it is sufficient to use a low order of approximation for the determination of the transport coefficients since higher orders contribute to the coefficients by a small percentage only. ${ }^{16}$ ) In URANUS different orders of approximation are employed depending on the coefficient under consideration. The highest order of approximation, namely $\alpha=3$, is used for the determination of the coefficient $a_{e 1}$ of the electrons. ${ }^{1)}$

In Eqs. (2) to (5), relations for determining mean gas properties have been given. Those can be reduced to equations of the type

$$
\overline{\Psi_{i}(\vec{x}, t)}=\int \Psi_{i}\left(\vec{c}_{i}\right) f_{i}\left(\vec{c}_{i}, \vec{x}, t\right) d \vec{c}_{i},
$$

where the value of the integral $\overline{\Psi_{i}(\vec{x}, t)}$ is denoted a moment of the velocity distribution function. An equation for the determination of $\overline{\Psi_{i}}$ is Maxwell's transport equation

$$
\begin{aligned}
& \int \Psi_{i}\left\{\frac{\partial f_{i}}{\partial t}+\vec{c}_{i} \cdot \nabla f_{i}+\frac{\vec{F}_{i}}{m_{i}} \cdot \frac{\partial f_{i}}{\partial \vec{c}_{i}}\right\} d \vec{c}_{i}= \\
& 2 \pi \int \Psi_{i}\left\{\sum_{j=1}^{z_{s}} \iint\left(f_{i}^{\prime} f_{j}^{\prime}-f_{i} f_{j}\right) g_{i j} b d b d \vec{c}_{j}\right\} d \vec{c}_{i},
\end{aligned}
$$

which can be derived by multiplication of Boltzmann's Equation (7) with the property $\overline{\Psi_{i}}$ and subsequent integration with respect to $\vec{c}_{i}$. Since the molecular property $\Psi_{i}$ is independent of space and time, one can rewrite Maxwell's transport equation and obtains

$$
\begin{aligned}
& \frac{\partial \overline{\Psi_{i}}}{\partial t}+\frac{\partial \overline{\Psi_{i} \vec{c}_{i}}}{\partial \vec{x}}+\frac{\vec{F}_{i}}{m_{i}}\left(\frac{\partial \overline{\Psi_{i}}}{\partial \vec{c}_{i}}-\frac{\overline{\partial \Psi_{i}}}{\partial \vec{c}_{i}}\right)= \\
& 2 \pi \sum_{j=1}^{z_{s}} \iiint \Psi_{i}\left(f_{i}^{\prime} f_{j}^{\prime}-f_{i} f_{j}\right) g_{i j} b d b d \vec{c}_{i} d \vec{c}_{j},
\end{aligned}
$$

employing partial integration. In the following the contribution of external forces is neglected. 
The collision integral becomes zero when evaluating Maxwell's transport equation for the collisional invariants. Those are mass, momentum and energy of the particles, i.e.

$$
\Psi_{i} \in\left\{m_{i}, m_{i} \vec{c}_{i}, \frac{1}{2} m_{i} c_{i}^{2}+m_{i} h_{0, i}+m_{i} e_{i n t, i}\right\} .
$$

The well known Euler Equations are obtained from Maxwell's transport equations using the maxwellian velocity distribution function (9) for $f$. If Maxwell's Transport Equation (16) is evaluated for the collision invariants from Eq. (17) with $f=f^{[1]}$ from Eq. (10) the Navier-Stokes Equations are obtained.

For $\Psi_{i}=m_{i}$ one finds

$$
\begin{aligned}
& \frac{\partial \rho_{i}}{\partial t}+\nabla^{T} \cdot\left[\rho_{i} \vec{v}-\frac{\rho_{i}}{2} \sqrt{\frac{2 k T_{i}}{m_{i}}} a_{i 0} \frac{\partial \ln T_{i}}{\partial \vec{x}}\right. \\
& \left.+\frac{n^{2}}{\rho} \sum_{j=1}^{z_{s}} m_{i} m_{j} \frac{\rho \psi_{i}}{2 m_{j}} \sqrt{\frac{2 k T_{i}}{m_{i}}} c_{i 0}^{(j, i)} \vec{d}_{j}\right]=S_{m, i}
\end{aligned}
$$

for the species balance equations. Employing the thermal diffusion coefficient

$$
D_{i}^{T}=\frac{\rho_{i}}{2} \sqrt{\frac{2 k T_{i}}{m_{i}}} a_{i 0}
$$

and the multi component diffusion coefficients

$$
D_{i j}=\frac{\rho \psi_{i}}{2 m_{j}} \sqrt{\frac{2 k T_{i}}{m_{i}}} c_{i 0}^{(j, i)}
$$

and introducing the mass diffusion flux by

$$
n_{i} m_{i} \overrightarrow{\vec{V}_{i}}=\vec{j}_{i}=\frac{n^{2}}{\rho} \sum_{j=1}^{z_{s}} m_{i} m_{j} D_{i j} \vec{d}_{j}-D_{i}^{T} \frac{\partial \ln T_{i}}{\partial \vec{x}}
$$

Eq. (18) can be simplified to

$$
\frac{\partial \rho_{i}}{\partial t}+\nabla^{T} \cdot\left[\rho_{i} \vec{v}+\vec{j}\right]=S_{m, i} .
$$

Note that the determination of the multi component diffusion coefficients is not well-defined since the conditional equations for determining the sonine coefficients $c_{i 0}^{(j, i)}$ are linearly dependent. Because of the fact that $\sum_{j} \vec{d}_{j}=\overrightarrow{0}$, one of the coefficients can be chosen arbitrarily. The physical meaning is that the coefficients $c_{i 0}^{(j, i)}$ depend on the choice of a reference speed. Following Chapman and Cowling ${ }^{14)}$ and Hirschfelder et al. ${ }^{13)}$ it is common to chose $\overrightarrow{\vec{V}}_{i}$ as the reference speed which is equivalent to $D_{i i}=c_{i 0}^{(i, i)}=0$. Within URANUS, ten species balance equations are solved for $\mathrm{N}_{2}, \mathrm{O}_{2}, \mathrm{NO}, \mathrm{N}, \mathrm{O}, \mathrm{N}_{2}^{+}, \mathrm{O}_{2}^{+}, \mathrm{NO}^{+}, \mathrm{O}^{+}$and $\mathrm{N}^{+}$. Based on the quasi neutral assumption, the electron number density is determined from the number density of the ionised species.

With $\Psi_{i}=m_{i} \vec{c}_{i}$ one obtains

$$
\begin{array}{r}
\frac{\partial\left(\rho_{i} \vec{v}\right)}{\partial t}+\nabla^{T} \cdot\left[\rho_{i} \vec{v} \vec{v}^{T}+n_{i} k T_{i} \overline{\bar{E}}+\vec{v} \vec{j}_{i}^{T}+\vec{j}_{i} \vec{v}^{T}\right. \\
\left.-\frac{1}{2} n_{i} k T_{i} b_{i 0} \overline{\bar{K}}\right]=\vec{S}_{I, i}
\end{array}
$$

for the momentum transport of the species, where

$$
\begin{aligned}
\overline{\bar{K}} & =\nabla \vec{v}^{T}+\left(\nabla \vec{v}^{T}\right)^{T}-\left(\frac{2}{3} \nabla^{T} \vec{v}\right) \overline{\bar{E}} \\
& =\left[\begin{array}{ccc}
2 \frac{\partial u}{\partial x}-\frac{2}{3} \nabla^{T} \vec{v} & \frac{\partial v}{\partial x}+\frac{\partial u}{\partial y} & \frac{\partial w}{\partial x}+\frac{\partial u}{\partial z} \\
\frac{\partial u}{\partial y}+\frac{\partial v}{\partial x} & 2 \frac{\partial v}{\partial y}-\frac{2}{3} \nabla^{T} \vec{v} & \frac{\partial w}{\partial y}+\frac{\partial v}{\partial z} \\
\frac{\partial u}{\partial z}+\frac{\partial w}{\partial x} & \frac{\partial v}{\partial z}+\frac{\partial w}{\partial y} & 2 \frac{\partial w}{\partial z}-\frac{2}{3} \nabla^{T} \vec{v}
\end{array}\right]
\end{aligned}
$$

is the rate of the shear stress tensor. Employing the thermal equation of state

$$
p=\sum_{\substack{i=1 \\ i \neq e^{-}}}^{z_{s}} \rho_{i} R_{i} T+\rho_{e} R_{e} T_{e}
$$

and the coefficient of viscosity

$$
\eta=\frac{1}{2} k \sum_{i=1}^{z_{s}} n_{i} T_{i} b_{i 0}
$$

the momentum conservation equation

$$
\frac{\partial(\rho \vec{v})}{\partial t}+\nabla^{T} \cdot\left[\rho \vec{v} \vec{v}^{T}+p \overline{\bar{E}}-\eta \overline{\bar{K}}\right]=\overrightarrow{0}
$$

follows, when summing up the species momentum equations for all species.

When solving Maxwell's Transport Equation (16) with $f=f^{[1]}$ for $\Psi_{i}=1 / 2 m_{i} c_{i}^{2}$, one obtains

$$
\begin{aligned}
\frac{\partial\left(\rho_{i} e_{t r, i}\right)}{\partial t}+\nabla^{T} \cdot\left[\frac{5}{2} \frac{k T_{i}}{m_{i}}\left(\rho_{i} \vec{v}+\vec{j}_{i}\right)+\vec{v} \vec{v}^{T} \cdot \vec{j}_{i}\right. \\
-\frac{1}{2} n_{i} k T_{i} b_{i 0} \overline{\bar{K}} \cdot \vec{v}+\frac{5}{8} \rho_{i}\left(\frac{2 k T_{i}}{m_{i}}\right)^{\frac{3}{2}} a_{i 1} \frac{\partial \ln T_{i}}{\partial \vec{x}} \\
\left.+\frac{v^{2}}{2}\left(\rho_{i} \vec{v}+\vec{j}_{i}\right)\right]=S_{t r, i}
\end{aligned}
$$

for the translational energy, where $e_{t r, i}=3 / 2 R_{i} T_{i}+$ $1 / 2 v^{2}$. In URANUS the translational thermal conductivity is distinguished between that of heavy particles

$$
\lambda_{t r}=-\frac{5}{4} k \sum_{\substack{i=1 \\ i \neq e^{-}}}^{z_{s}} n_{i} \sqrt{\frac{2 k T}{m_{i}}} a_{i 1}
$$

and that of electrons

$$
\lambda_{t r, e}=-\frac{5}{4} k n_{e} \sqrt{\frac{2 k T_{e}}{m_{e}}} a_{e 1} .
$$

The transport coefficients defined in Eqs. (19), (20), (26), (29) and (30) are computed as explained in a previous publication. ${ }^{1)}$ Note that the models account only for elastic collisions for the determination of the transport coefficients. As in most other codes, the influence of inelastic collisions is considered only for the determination of the source terms, while the influence on the transport coefficients is neglected, see e.g. Graves and Argrow. ${ }^{17}$ ) 
With the stress tensor

$$
\overline{\bar{\tau}}=\eta \overline{\bar{K}}
$$

and Eqs. (31), (29) and (30), the translational energy balance equation can be expressed by

$$
\begin{gathered}
\frac{\partial\left(\rho e_{t r}\right)}{\partial t}+\nabla^{T} \cdot\left[\frac{\rho v^{2}}{2} \vec{v}+\sum_{\substack{i=1 \\
i \neq e^{-}}}^{z_{s}}\left(\frac{5}{2} R_{i} T\left(\rho_{i} \vec{v}+\vec{j}_{i}\right)\right)\right. \\
+\left(\frac{5}{2} R_{e} T_{e}\left(\rho_{e} \vec{v}+\vec{j}_{e}\right)\right)-\overline{\bar{\tau}} \cdot \vec{v} \\
\left.-\lambda_{t r} \frac{\partial T}{\partial \vec{x}}-\lambda_{t r, e} \frac{\partial T_{e}}{\partial \vec{x}}\right]=S_{t r}
\end{gathered}
$$

Since the formation enthalpy is a component specific constant independent of velocity, the species formation energy balance equation

$$
\frac{\partial\left(\rho_{i} h_{0, i}\right)}{\partial t}+\nabla^{T} \cdot\left[\rho_{i} h_{0, i} \vec{v}+h_{0, i} \vec{j}\right]=S_{c h, i}
$$

which is obtained for $\Psi_{i}=m_{i} h_{0, i}$, has the same form as the species mass balance equations. In URANUS the source term $S_{c h, i}=S_{m, i} h_{0, i}$ is considered in the caloric equation of state. Due to chemical reactions, the thermal energy content of the flow changes. For physical and chemical processes usually only the energy differences are of interest. For a general description a reference has to be defined. In chemistry the standard state of the elements of the periodic table is defined by $T^{0} \equiv 25^{\circ} \mathrm{C}=298,15 \mathrm{~K}$ and $p^{0} \equiv 1 \mathrm{~atm}=101300 \mathrm{~Pa}$. In addition, the standard enthalpy of formation for an element in its standard state, i.e. the most common condition of state, is zero. In the case of URANUS, which considers the elements Nitrogen and Oxygen, the standard states are the molecular species $\mathrm{N}_{2}$ and $\mathrm{O}_{2}$. Based on the definition of the standard state, the standard enthalpy of formation for most compounds can be found in literature. ${ }^{18,19)}$ Since URANUS works according to the Kelvin scale, the standard formation enthalpy has to be converted according to

$$
h_{0, i}=\frac{\Delta H_{f, i}^{0}}{M_{i}}-\int_{0}^{298,15} c_{p, i}(T) d T .
$$

In addition to formation enthalpy, the species are characterised by additional energy fractions due to rotation, vibration and electronic excitation, which is caused by the bound electrons of the chemical species in electron shells above the ground level. All currently available models for CFD follow the Born-Oppenheimer approximation, which states that rotation, vibration and electronic excitation are not coupled. If the internal energy states are decoupled, the internal energy of a molecule can be determined from the superposition of rotation, vibration and electronic energy

$$
\varepsilon_{i n t}=\varepsilon_{r o t}(J)+\varepsilon_{v i b}(v)+\varepsilon_{e l}(l),
$$

where $\varepsilon_{\text {int }}$ is the energy difference between ground state and actual quantum state. From a classical point of view, electronic excitation tends to lower the binding energy between molecular species. Hence, the internuclear distance increases, which in turn influences vibration. Changes of vibration alter the moment of inertia of a molecule and, therefore, influence rotation. However, several simplifications that are expected to have a more significant effect on flow properties than the Born-Oppenheimer approximation will be described in the following.

In state selective models, the different energy content is considered to distinguish between species. The additional energy fraction is added to the formation enthalpy. Each change of the internal quantum state is handled like a chemical reaction. Unfortunately, molecular species have a vast number of internal energy states. If one assumes 10 electronic levels with 30 vibrational and 300 rotational levels, a number of roughly 100000 levels arises. Even if levels with similar energy are grouped, a high number of species follows. The major disadvantage of the state selective approach is the extraordinary effort for determining the diffusion velocities and the reaction rates, which disallows for the use of state selective models for most real gas flow problems of interest. Hence, simplifications are necessary. The determination of the diffusion velocities becomes feasible under the assumption that the collision cross sections of the components are independent of quantum state. Hence, the diffusion coefficients of all species that differ only by quantum state are equal, which significantly facilitates the computation of the diffusion velocities. A more constrictive approach can be derived from equilibrium considerations. In equilibrium, the population density of the quantum states $k$ can be described by analytical functions

$$
f_{\text {int }, i}\left(k, T_{i n t, i}\right)=\frac{n_{k}}{n_{i}} \quad \text { with } \quad n_{i}=\sum_{k=1}^{k_{\max }} n_{k},
$$

which depend on only one distribution parameter $T_{i n t, i}$. Reasonably, the distribution parameter $T_{i n t, i}$ is defined such that in equilibrium $T=T_{\text {int }, i}$ holds. This also applies if the deviation from equilibrium is small. With increasing nonequilibrium the temperatures differ, but the assumption of an analytical distribution function still holds if nonequilibrium is not too strong, which is the basis of the multi-temperature models. In contrast to the state selective approach where species balance equations for all quantum states have to be solved, the conditional equations of the multi-temperature approach are energy balance equations. The energy fraction associated with the excitation of internal degrees of freedom is linked to the distribution parameter $T_{\text {int }, i}$ by

$$
e_{i n t, i}\left(T_{i n t, i}\right)=\frac{1}{m_{i}} \sum_{k=1}^{k_{\max }} f_{i n t, i}\left(k, T_{i n t, i}\right) \varepsilon_{\text {int }, k},
$$

where $k$ is an arbitrary quantum state.

For the basic principles of multi-temperature approach it is not necessary to distinguish between rotational, vibrational and electronic excitation since the treatment is 
identical for all types of internal excitation. In order to determine the distribution parameter $T_{i n t, i}$, one may form two transport equations of the types of Eqs. (22) and (33) under consideration of Eq. (36) for each of the quantum states. Subsequently, the equations for one chemical species are summed up. A difficulty arises from the first term of the gradient vector for diffusion (13). From the chain rule follows

$$
\begin{array}{r}
\nabla^{T} \phi_{k}=\nabla^{T}\left(\frac{n_{k} k T_{i}}{p}\right)=\nabla^{T}\left(f_{\text {int }, i} \phi_{i}\right) \\
=f_{\text {int }, i} \nabla^{T} \phi_{i}+\phi_{i} \nabla^{T} f_{\text {int }, i}
\end{array}
$$

which contains an additional diffusion contribution from $\phi_{i} \nabla^{T} f_{i n t, i}$ due to the spacial dependency of the internal distribution function. Hence, the modelling of diffusion in the multi-temperature approach becomes as challenging as in the state selective approach. In order to approximate the influence of the additional diffusion flux, it is determined for a homogeneous gas. Under the assumption that the collision cross sections are independent of quantum state, the transport equations

$$
\begin{aligned}
& \frac{\partial\left(n_{k} \varepsilon_{i n t, k}\right)}{\partial t} \\
& +\nabla^{T}\left(n_{k} \varepsilon_{i n t, k}\left[\vec{v}-\frac{n}{n_{k}} \mathscr{\mathscr { D }} \frac{\partial}{\partial \vec{x}}\left(\frac{n_{k}}{n}\right)\right]\right)=S_{i n t, k}
\end{aligned}
$$

for the internal energy transport of the quantum states follow. Since $\varepsilon_{i n t, k}$ is independent of time and space, the subsequent summation can be exchanged with the differentiation. One obtains

$$
\begin{aligned}
\sum_{k=1}^{k_{\max }}\left\{\varepsilon_{i n t, k} n \mathscr{\mathscr { D }} \frac{\partial}{\partial \vec{x}}\left(\frac{n_{k}}{n}\right)\right\} & =n \mathscr{\mathscr { D }} \frac{\partial}{\partial \vec{x}}\left(\sum_{k=1}^{k_{\max }} \varepsilon_{i n t, k} f_{i n t, k}\right) \\
& =\rho \mathscr{D} \frac{\partial e_{i n t}\left(T_{i n t}\right)}{\partial T_{i n t}} \frac{\partial T_{i n t}}{\partial \vec{x}}
\end{aligned}
$$

for the additional diffusion term under consideration of Eq. (37). Hence, the influence of the additional diffusion flux can be treated as an additional contribution to heat conduction. Employing the specific heat capacity for the internal excitation

$$
c_{v, i n t}\left(T_{i n t}\right)=\frac{\partial e_{i n t}\left(T_{i n t}\right)}{\partial T_{i n t}},
$$

the associated internal heat conductivity

$$
\lambda_{\text {int }}=n m \mathscr{D} c_{v, \text { int }}\left(T_{\text {int }}\right)
$$

can be determined. One finally gets

$$
\begin{aligned}
& \frac{\partial\left(\rho_{i} e_{i n t, i}\right)}{\partial t} \\
& +\nabla^{T} \cdot\left[e_{i n t, i}\left(\rho_{i} \vec{v}+\vec{j}_{i}\right)-\lambda_{i n t, i} \frac{\partial T_{i n t, i}}{\partial \vec{x}}\right]=S_{i n t, i}
\end{aligned}
$$

for the transport of internal energy, which serves as a conditional equation for the determination of the internal energy $e_{\text {int }, i}$ and $T_{\text {int }, i}$.

In URANUS Brokaw's approximation ${ }^{20)}$ is employed to convert the internal heat conductivity in a homogeneous gas to the internal heat conductivity in a gas mixture $\lambda_{i n t, i}$. Note that URANUS only considers the contribution of rotation and vibration, while electronic excitation is neglected in the current version. The energy conservation equation

$$
\begin{aligned}
& \frac{\partial\left(\rho_{i} e_{t o t}\right)}{\partial t} \\
& +\nabla^{T} \cdot\left[\rho h_{t o t} \vec{v}+\vec{q}+\sum_{i=1}^{z_{s}} h_{t o t, i} \vec{j}_{i}-\overline{\bar{\tau}} \cdot \vec{v}\right]=-S_{r a d}
\end{aligned}
$$

is found summing up all translational and internal contributions, where the total energy and the total enthalpy are

$$
\begin{aligned}
& \rho e_{t o t}=\frac{1}{2} \rho v^{2}+\sum_{\substack{i=1 \\
i \neq e^{-}}}^{z_{s}} \rho_{i}\left(\frac{3}{2} R_{i} T+h_{0, i}\right) \\
& +\sum_{j=1}^{z_{m o l}} \rho_{j} e_{v i b, j}+\sum_{k=1}^{z_{m o l}} \rho_{k} e_{r o t, k}+\rho_{e}\left(\frac{3}{2} R_{e} T_{e}+h_{0, e}\right)
\end{aligned}
$$

and

$$
\rho h_{t o t}=\rho e_{t o t}+p,
$$

respectively, and the convective heat flux vector is

$$
\begin{aligned}
\vec{q}=-\lambda_{t r} & \frac{\partial T}{\partial \vec{x}}-\lambda_{t r, e} \frac{\partial T_{e}}{\partial \vec{x}} \\
& -\sum_{i=1}^{z_{m o l}}\left(\lambda_{v i b, i} \frac{\partial T_{v i b, i}}{\partial \vec{x}}+\lambda_{r o t, i} \frac{\partial T_{r o t, i}}{\partial \vec{x}}\right) .
\end{aligned}
$$

Note that the only source term contribution to the total energy conservation equation is the radiation source term $S_{\text {rad }}$, since radiation generation and transport are not treated in the CFD code. For the simulation of hyperbolic entry flows, the radiation source term is computed with the radiation transport code HERTA (High Enthalpy Radiation Transport Algorithm) ${ }^{21}$ ), which employs the program PARADE (Plasma Radiation Database) to determine the gas radiation spectrally resolved. ${ }^{4,22)}$

Eqs. (22), (27), (28), (43) and (44) can be expressed by

$$
\partial \vec{Q} / \partial t+\nabla^{T} \cdot\left(\overrightarrow{\mathcal{F}}-\overrightarrow{\mathcal{F}}_{v}\right)=\vec{S} .
$$

The conservation vector

$$
\begin{aligned}
\vec{Q}= & \left(\rho_{i}, \rho u, \rho v, \rho e_{t o t}, \rho_{j} e_{v i b, j}, \sum_{k} \rho_{k} e_{r o t}, k, \rho_{e} e_{e}\right)^{T} \\
& (i=1, . ., 10, j=1, . ., 3, k=1, . ., 3,6, . ., 8)
\end{aligned}
$$

of the 2D/axisymmetric code consist of 10 species balance equations, two momentum equations, the total energy 
equation, 3 vibrational energy equations for the molecular species $\mathrm{N}_{2}, \mathrm{O}_{2}$ and $\mathrm{NO}$, a rotational energy equation for the molecules, and an electron energy equation. The source term vector accounts for chemical reactions as well as energy exchange due to radiation, inelastic collisions, and due to collisions between electrons and heavy particles. Thermochemical relaxation processes in the gas-phase are accounted for by the advanced multiple temperature Coupled Vibration-Chemistry-Vibration (CVCV) model ${ }^{3)}$. The influence of vibrational and rotational excitation on chemistry and the influence of chemical reactions on vibration and rotation are modelled consistently in the source terms of the conservation equations. In dissociated re-entry, flows strong gradients are observed in densities, temperatures, and velocities.

The discretization of the inviscid fluxes of the governing equations is performed in the physical space by a Godunov-type upwind scheme. $\left.{ }^{23}, 24\right)$ Second order accuracy is achieved by employing TVD or WENO limited extrapolation. ${ }^{2,5)}$ The upwind scheme constitutes a Riemann problem at the cell faces. High accuracy can be obtained with an exact linearisation of the Riemann problem. An approximate Riemann Solver for this approach has been developed by Roe ${ }^{24)}$. One of the problems associated with the linearisation of the equations is that the change from subsonic to supersonic flow is the direct inversion of the change from supersonic to subsonic flow. Hence, the scheme allows for unphysical rarefaction shocks that are associated with a decrease of entropy. In order to solve this problem according to the second main clause of thermodynamics, an entropy correction has to be applied. ${ }^{25}$ ) The entropy fix applied in URANUS suppresses both the occurrence of rarefaction shocks as well as the carbuncle phenomenon. ${ }^{2}$ ) In addition to the approximate Riemann Solver of Roe, two additional flux splitting schemes are implemented in URANUS. ${ }^{23,26)}$ Both schemes are very stable and are associated with less computational cost than the Roe solver, but are considered less accurate. Therefore, Roe's approximate solver has been employed for the determination of all results presented in section 3 . The viscous fluxes are discretized in the transformed computational space by central differences on structured grids using formulae of second order accuracy.

To calculate the steady state solution of the finite volume Navier-Stokes equations from Eq. (48)

$$
V(\partial \vec{Q} / \partial t)=\vec{R}(\vec{Q})
$$

with the sum of the inviscid and viscous fluxes and the source terms $\vec{R}(\vec{Q})$, the implicit Euler time differencing with the usual Taylor series linearisation for $\vec{R}$ is applied. The resulting linear system

$$
[V / \Delta t-(\partial \vec{R} / \partial \vec{Q})] \Delta \vec{Q}=\vec{R}(\vec{Q})
$$

with $\vec{Q}^{n+1}=\vec{Q}^{n}+\Delta \vec{Q}$, has to be solved for each time step. For $\Delta t \rightarrow \infty$ the scheme is exactly Newton's method.
Heterogenous surface reactions on the TPS influence the surface heat flux significantly. Kinetic gas-surface interaction models are implemented in the URANUS code in order to take into account near-surface rarefaction effects in leeward flows, base flows, and general re-entry flows at high altitudes. ${ }^{6,11)}$ Mass, momentum and energy fluxes to the TPS are computed by solving Maxwell's transport equation (15) for the particles approaching the surface. Surface slip is modelled by balancing incoming and outgoing fluxes at the surface. The global catalysis model, which assumes complete chemical energy accommodation at the surface, is presently used in the 3D version of the URANUS code.9) The catalytic behavior of the implemented technical surfaces $\left(\mathrm{SiC}, \mathrm{SiO}_{2}\right)$ is modelled by overall recombination coefficients which were measured by Stewart in a large surface temperature range. ${ }^{27)}$ For design approaches, nonand fully-catalytic cases can also be simulated by the $3 \mathrm{D}$ URANUS code. More advanced gas-surface interaction models, which allow for a detailed simulation of the elementary reactions as well as of active and passive oxidation of $\mathrm{SiC}$, are available in the 2D/axisymmetric code. ${ }^{8,11,10)}$ The 3D Parallel-Multiblock URANUS code features models for radiative exchange between the surface elements and for heat conduction within the TPS. ${ }^{28)}$ Most of the advanced complex surface boundary conditions as well as all other boundary conditions are implemented implicitly.

\section{Results}

In order to invesitgate the influence of the slip flow modelling, simulations of the re-entry of the US Space Shuttle orbiter at high altitudes have been performed. ${ }^{11,12)}$ The results have been compared to Navier-Stokes ${ }^{6}$ ) and DSMC ${ }^{29,30)}$ results at altitudes of $92.35 \mathrm{~km}, 99.49 \mathrm{~km}, 105.31 \mathrm{~km}$ and at $109.75 \mathrm{~km}$, where significant rarefaction effects arose. In order to compare the results, no reactions have been considered at the surface and complete accommodation was assumed. The axisymmetric simulations have been performed for equivalent hyperboloids on structured grids consisting of $89 \times 76$ cells.

For the $92.35 \mathrm{~km}$ altitude case good agreement of the shock stand-off distance and the post shock behaviour of translational temperature compared to the DSMC result of Carlson and Bird ${ }^{29}$ ) is shown in Fig. 1. The agreement in vibrational temperature is rather poor, which is assumed to originate from different gas-phase relaxation models. The skin friction coefficients determined with slip model are shown in Fig. 2. When comparing with the DSMC results of Moss and Bird ${ }^{30)}$, good agreement can be observed.

Fig. 3 compares the heat fluxes with applied slip boundary conditions to the DSMC results of Moss and Bird ${ }^{30}$. Surprisingly, disagreement is found especially at low altitudes, where both Navier-Stokes as well as DSMC approach are expected to obtain equal results. As found by 7) the differences arise from the different post shock relaxation models and do not indicate a failure of the NavierStokes equations.

The influence of the catalysis and radiative transfer on 


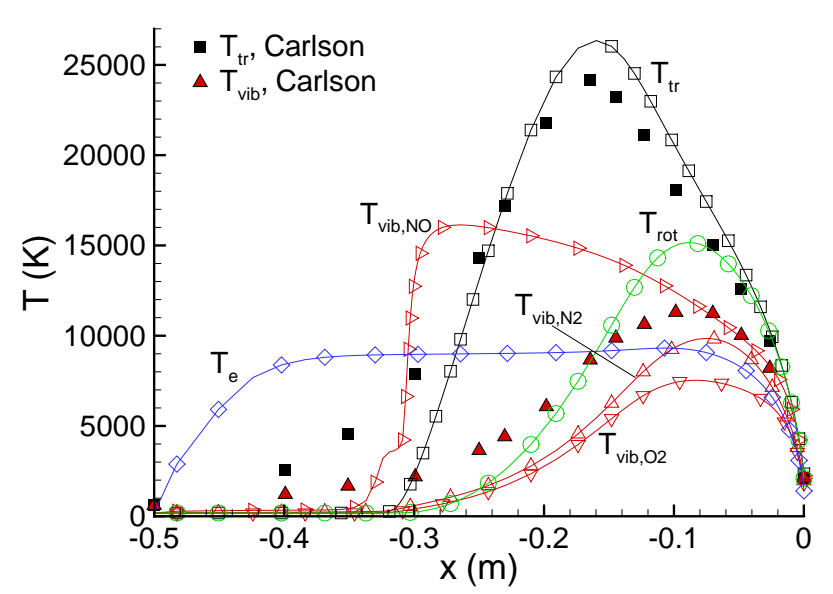

Fig. 1. Comparison of the simulated temperature distribution versus distance from stagnation point ${ }^{11,12)}$ with the DSMC results of Carlson ${ }^{29)}$.

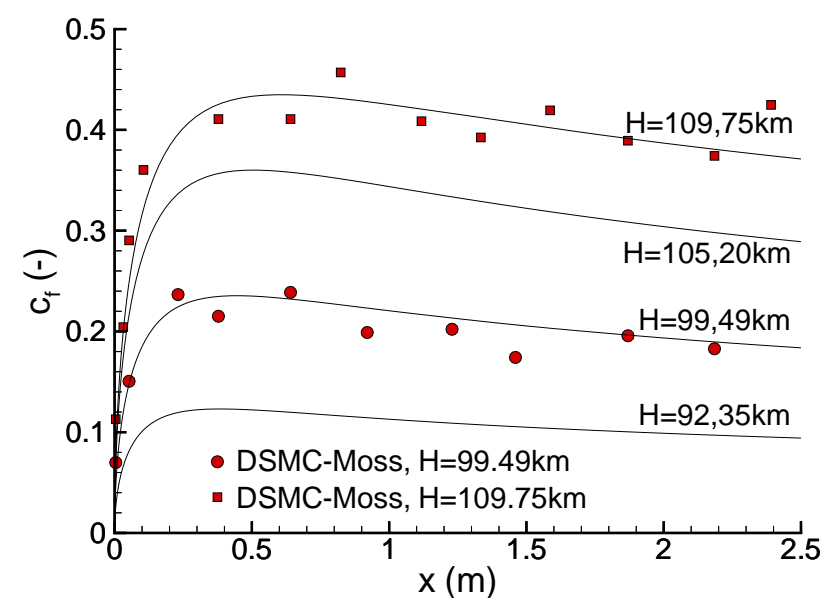

Fig. 2. Comparison of skin friction coefficients computed with slip boundary conditions ${ }^{11,12)}$ with DSMC results of Moss and Bird $^{30)}$.

the thermal loads of a re-entry vehicle have been investigated for the re-entry of the X-38 vehicle at $64.6 \mathrm{~km}$ of altitude and a velocity of $6085 \mathrm{~m} / \mathrm{s}^{31}$ ) The X-38 experimental vehicle was developed as a prototype for the Crew Return Vehicle (CRV), which was intended to allow for a quick return of astronauts on the International Space Station to earth in cases of emergency. The results of X-38 are of particular interest for the modelling of heat conduction within the TPS and radiative exchange at the surface, since the geometry of the vehicle has concave shapes and angular parts. In order to withstand the thermal loads during re-entry, the vehicle was equipped with different thermal protection systems according to the expected loads. Nose cap, nose skirt, and chin panel are made of $\mathrm{C} / \mathrm{C}-\mathrm{SiC}$, which is considered in the simulations. The rest of X-38's surface is equipped with $\mathrm{SiO}_{2}$ tiles, which is also taken into account for the simulations discussed here. At the considered trajectory point, X-38 has an angle of attack of $\alpha=40^{\circ}$ and the body flap deflection angle is $\delta=20^{\circ}$. For the simulations of the X-38, a multiblock mesh from DLR has been

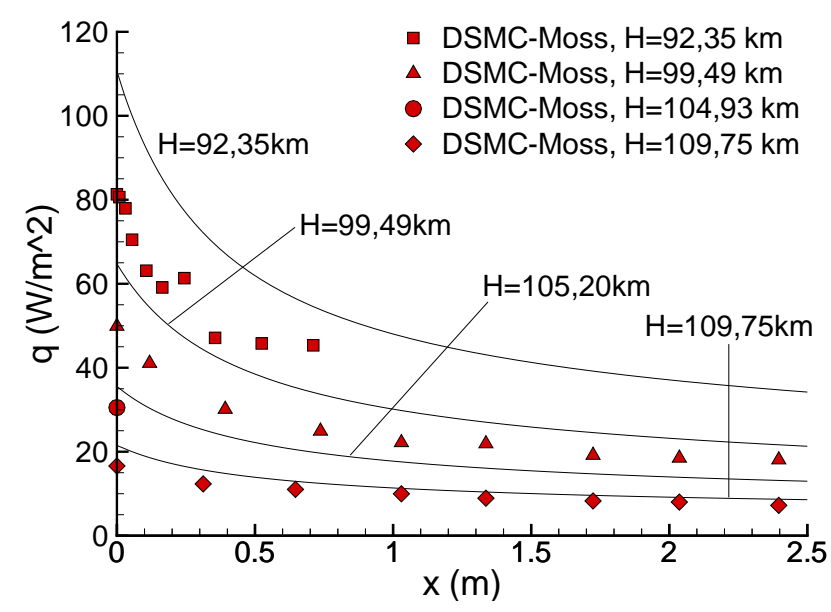

Fig. 3. Comparison of surface heat flux computed with the flux based slip boundary conditions ${ }^{11,12)}$ with DSMC results of Moss and $\operatorname{Bird}^{30)}$.

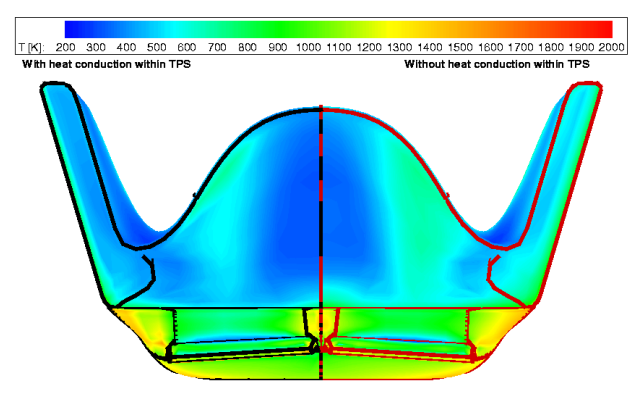

Fig. 4. Temperature difference at the cavity region of the body flap of the X-38 due to heat conduction within the TPS. ${ }^{31}$ )

used, which contains wake flow regions. The mesh has 1.02 million cells and consists of 18 initial blocks. The resolution in the normal direction is 80 cells. For the tangential directions, the mesh is resolved by 66 cells for each initial block. The SiC-based tiles of the X-38's TPS have a thickness of $4 \times 10^{-3} \mathrm{~m}$. For simplification the $\mathrm{SiO}_{2}$-based tiles were approximated as having the same thickness. The TPS grid consists of finite linear brick elements and is resolved by 20 cells in the orthogonal direction. The resolution in tangential directions is the same as for the flow mesh.

Fig. 4 shows the influence of TPS heat conduction on the surface temperature distribution at the rear side. At first sight there are no great differences to observe; the temperature distribution has the same topology. However, the temperature distribution is smoothed. The temperature displacements show a 3D behavior with significant changes in the maximum values varying between $-100 \mathrm{~K}$ and $+200 \mathrm{~K}$. As shown in Fig. 5, a significant increase of the surface temperature has been observed at the cavity zone of the vehicle's body flap where the radiative exchange is a dominant mechanism. The simulations depicted an increase of $500 \mathrm{~K}$ at the main body of the $\mathrm{X}-38$ and $370 \mathrm{~K}$ at the body flap. ${ }^{31)}$ This result is very important and shows how significant the consideration of radiative exchange is. Considering the low pressure in this concave zone, active oxidation of the $\mathrm{C} / \mathrm{SiC}$ 


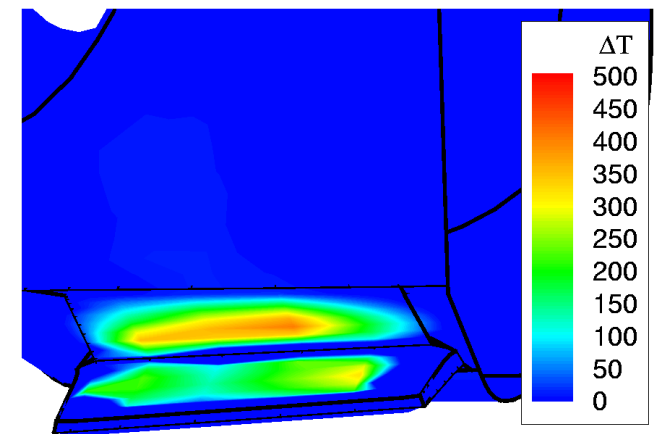

Fig. 5. Temperature difference at the cavity region of the body flap of the $\mathrm{X}-38$ due to radiative exchange. ${ }^{31)}$

material would have been highly probable.

Ceramics based on $\mathrm{SiC}$ withstand temperatures above $2000 \mathrm{~K}$ and have a high emissivity, which allows for an effective radiation cooling of the surface. Hence, the nose caps of the US Shuttle orbiter, X-38 and Hope-X are all based on silicon carbide. In comparison to $\mathrm{SiO}_{2}$-based materials, the catalysis of $\mathrm{SiC}$ concerning Oxygen and Nitrogen atoms is significantly higher at high temperatures. 27) Furthermore, $\mathrm{SiC}$ may react with Oxygen or Nitrogen, forming the gaseous species $\mathrm{SiO}, \mathrm{SiN}, \mathrm{CO}$ and $\mathrm{CN}$. If the surface temperature is sufficiently low and the oxygen partial pressure is sufficiently high, a solid $\mathrm{SiO}_{2}$-layer may form at the surface and acts as a protection layer for the underlying $\mathrm{SiC}$. All of the reactions described so far are exothermal, i.e. chemical energy is transferred towards the surface. Therefore, a protective $\mathrm{SiO}_{2}$-layer is desirable at the surface since $\mathrm{SiO}_{2}$ not only protects the $\mathrm{SiC}$ from further oxidation but is also less catalytic. Ambient conditions leading to the formation of a protective $\mathrm{SiO}_{2}$-layer are called 'passive'. Unfortunately, the protective $\mathrm{SiO}_{2}$-layer is removed from the surface in the temperature range of $1600 \mathrm{~K}$ to $2100 \mathrm{~K}$ depending on oxygen partial pressure. As a consequence, the bare $\mathrm{SiC}$ is exposed to the highly reactive, partially dissociated gas flow. In this case, the reaction behavior is called 'active'.

The influence of active and passive oxidation on surface loads on $\mathrm{SiC}$ based materials has been investigated for the MIRKA re-entry flow under conditions where active as well as passive oxidation of $\mathrm{SiC}$ is possible. ${ }^{12)}$ MIRKA is a German experimental re-entry capsule, with a spherical shape of $1 \mathrm{~m}$ in diameter that was successfully flown in October 1997. To protect MIRKA against thermal loads during the re-entry, the outer layer of the TPS consisted of a C/SiC heat shield. The surface model for $\mathrm{SiC}$ developed by Fertig et al. ${ }^{10,12)}$ accounts for active and passive oxidation of $\mathrm{SiC}$ as well as for catalysis on $\mathrm{SiO}_{2}$ and $\mathrm{SiC}$. The results of the simulations have been compared with the experimtal results of the HEATIN (Heat Shield Instrumentation) experiment. ${ }^{32}$ ) Under peak heating conditions at about $56 \mathrm{~km}$ of altitude and a velocity of $6626 \mathrm{~m} / \mathrm{s}$, the gas conditions were transitional in the stagnation area, i.e. both active as well as passive oxidation of $\mathrm{SiC}$ was possible. Hence, oxidation at the surface depends on the initial oxidation state.
In order to investigate the influence of the transition on surface heat flux, URANUS was firstly initiated with passive oxidation (PAT study) and secondly initiated with active oxidation (APT study). The resulting heat fluxes at the

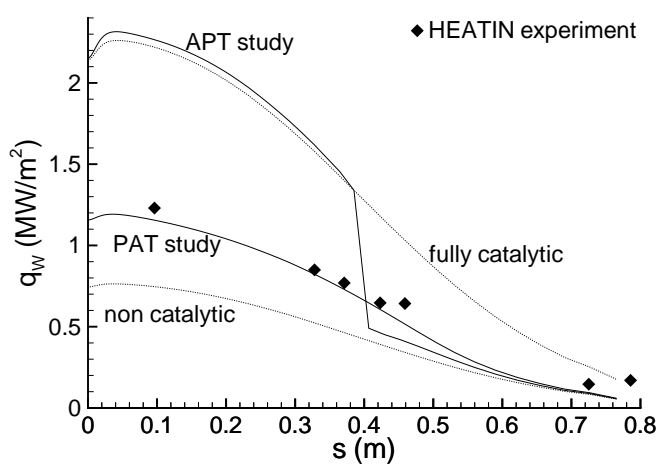

Fig. 6. Surface heat flux distribution along MIRKA's surface for the APT and PAT transition studies as well as for the fully and the non catalytic surface assumption in comparison to the results of the HEATIN experiment.

surface are shown in Fig. 6 together with the results for the non catalytic and the fully catalytic surface assumption in comparison to the experimental results of HEATIN. When initialised with active oxidation (APT study) the surface model predicted a transition to passive oxidation at $s \geq 0,4 \mathrm{~m}$. Note that the heat flux resulting from active oxidation exceeds the fully catalytic heat flux which is usually considered a worst case design load. No transition was observed within the PAT study where URANUS was initialised with passive oxidation. As can be seen from Fig. 6, the heating results of the PAT study agree very good with the results of the HEATIN experiment. A further significant

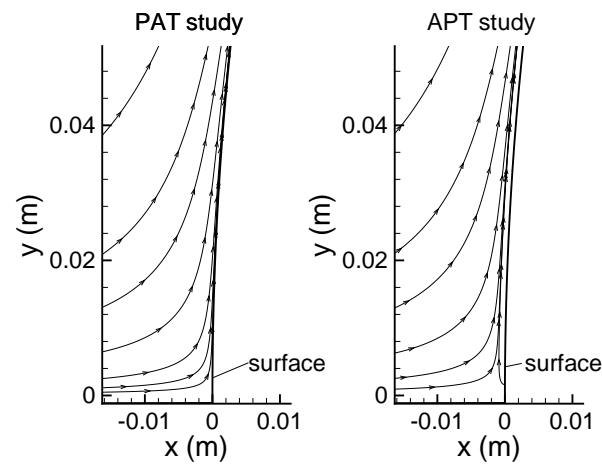

Fig. 7. Comparison of the stream line distributions in MIRKA's stagnation area resulting from the APT and the PAT transition studies.

finding of the PAT and the APT studies is shown in Fig. 7 where the stream lines in the stagnation area are shown. Active oxidation is associated with a rapid material loss at the surface. As a result, the stream lines originate at the surface in an active oxidation environment. 


\section{Summary}

Within this paper the core of the URANUS nonequilibrium has been explained based on rigorous kinetic theory.

By comparison of simulation results with other codes and experimental data the potential of URANUS has been demonstrated.

Future work is intended to focus on atmospheres of other celestial bodies and the modelling of electronic excitation.

\section{Acknowledgements}

The authors thank the Deutsche Forschungsgemeinschaft (DFG), the German Aerospace Center DLR and the federal state Baden-Württemberg for their support.

\section{References}

1) Fertig, M., Dohr, A. and Frühauf, H.-H.: Transport Coefficients for High Temperature Nonequilibrium Air Flows, AIAA Journal of Thermophysics and Heat Transfer, 15 (2001), pp. 148-156.

2) Jonas, S., Messerschmid, E., Frühauf, H.-H. and Knab, O.: Development and Validation of a High-Resolution Shock-Capturing Scheme for Nonequilibrium Hypersonic Flows, in Thirteenth International Conference on Numerical Methods in Fluid Dynamics, Springer, Berlin / Heidelberg, Germany, 1993, Vol. 414 of Lecture Notes in Physics, pp. 325-329, ISBN 978-3-540-56394-5.

3) Kanne, S., Knab, O., Frühauf, H.-H. and Messerschmid, E.: The Influence of Rotational Excitation on Vibration-Chemistry-VibrationCoupling, AIAA-Paper 96-1802, 1996.

4) Kanne, S.: Zur thermo-chemischen Relaxation innerer Freiheitsgrade durch Stoß- und Strahlungsprozesse beim Wiedereintritt, Ph.D. thesis, Institut für Raumfahrtsysteme, Universität Stuttgart, Germany, 2000, (in German).

5) Rödiger, T.: Analyse limitierter Extrapolationsverfahren zur Rekonstruktion von Hyperschallströmungen im thermochemischen Nichtgleichgewicht, Diplomarbeit IRS 04-S-06, Institut für Raumfahrtsysteme, Universität Stuttgart, Germany, 2004, (in German).

6) Daiß, A., Frühauf, H.-H. and Messerschmid, E.: New Slip Model for the Calculation of Air Flows in Chemical and Thermal Nonequilibrium, in Hunt, J. J., ed., Aerothermodynamics for Space Vehicles, 1995, Vol. 367 of ESA Special Publication, pp. 155-162.

7) Daiß, A.: Modellierung heterogener Reaktionen auf Siliziumdioxidoberflächen unter Berücksichtigung von Verdünnungseffekten, $\mathrm{Ph} . \mathrm{D}$. thesis, Institut für Raumfahrtsysteme, Universität Stuttgart, Germany, 1997, (in German).

8) Daiß, A., Frühauf, H.-H. and Messerschmid, E.: Modeling of Catalytic Reactions on Silica Surfaces with Consideration of Slip Effects, Journal of Thermophysics and Heat Transfer, 11 (1997), pp. 346352.

9) Fertig, M. and Frühauf, H.-H.: Detailed Computation of the Aerothermodynamic Loads of the MIRKA Capsule, in Harris, R. A., ed., Aerothermodynamics for Space Vehicles, 1999, Vol. 426 of ESA Special Publication, pp. 703-710.

10) Fertig, M., Frühauf, H.-H. and Auweter-Kurtz, M.: Modelling of Reactive Processes at SiC Surfaces in Rarefied Nonequilibrium Airflows, AIAA-Paper 2002-3102, 8th AIAA Joint Thermophysics and Heat Transfer Conference, St. Louis, Missouri, USA, 2002.

11) Fertig, M. and Auweter-Kurtz, M.: Flux Based Boundary Conditions for Navier-Stokes Simulations, in Danesy, D., ed., Fifth European Symposium on Aerothermodynamics for Space Vehicles, 2005, Vol. 563 of ESA Special Publication, pp. 501-508.

12) Fertig, M.: Modellierung reaktiver Prozesse auf SiliziumkarbidOberflächen in verdünnten Nichtgleichgewichts-Luftströmungen, Ph.D. thesis, Universität Stuttgart, Stuttgart, Germany, URN: urn:nbn:de:bsz:93-opus-24683, 2005, (in German), URL http://elib.uni-stuttgart.de/opus/volltexte/ $2005 / 2468 /$.

13) Hirschfelder, J., Curtiss, C. and Bird, R.: Molecular Theory of Gases and Liquids, John Wiley \& Sons, New York, 1954.

14) Chapman, S. and Cowling, T.: The Mathematical Theory of NonUniform Gases, Cambridge University Press, 3rd edn., 1970.

15) Enskog, D.: Kinetische Theorie der Vorgänge in mäßig verdünnten Gasen, Ph.D. thesis, Universität Uppsala, 1917, (in German).

16) Assael, M., Wakeham, W. and Kestin, J.: Higher-Order Approximation to the Thermal Conductivity of Monatomic Gas Mixtures, International Journal of Thermophysics, 1 (1980), pp. 7-32.

17) Graves, R. and Argrow, B.: Bulk Viscosity: Past to Present, Journal of Thermophysics and Heat Transfer, 13 (1999), pp. 337-342.

18) Cox, J., Wagman, D. and Medvedev, V.: CODATA Key Values for Thermodynamics, Hemisphere Publishing Corp., New York, 1989.

19) National Institute of Standards and Technology: NIST Chemistry WebBook, URL http: / / webbook.nist.gov/chemistry/.

20) Brokaw, R.: Approximate Formula for the Viscosity and Thermal Conductivity of Gas Mixtures, The Journal of Chemical Physics, 29 (1958), pp. 391-397.

21) Gogel, T., Dupuis, M. and Messerschmid, E.: Radiation Transport Calculation in High Enthalpie Environments for Two-Dimensional Axisymetric Geometries, Journal of Thermophysics and Heat Transfer, 8 (1994), pp. 744-750.

22) Winter, M., Pfeiffer, B., Fertig, M., Auweter-Kurtz, M. and Smith, A.: Recent Status of the Plasma Radiation Database PARADE, AIAAPaper 2004-2376, 2004.

23) Chou, S. and Baganoff, D.: Kinetic Flux Vector Splitting for the Navier Stokes Equations, Journal of Computational Physics, 130 (1997), pp. 217-230.

24) Roe, P.: Approximate Riemann Solvers, Parameter Vectors and Difference Scheme, Journal of Computational Physics, 43 (1981), pp. 357-372.

25) Harten, A.: High Resolution Schemes for Hyperbolic Conservation Laws, Journal of Computational Physics, 49 (1983), pp. 357-393.

26) Eberle, A.: A Nonlinear Flux Vector Split Defect Correction Scheme for Fast Solutions of the Euler and Navier-Stokes Equations, in Proceedings of the $8^{\text {th }}$ International Conference on Hyperbolic Problems, Magdeburg, Germany, 2000, pp. 327-336, ISBN 3-7643-67113.

27) Stewart, D.: Determination of Surface Catalytic Efficiency for Thermal Protection Materials - Room Temperature to Their Upper Use Limit, AIAA-Paper 96-1863, $31^{\text {st }}$ Thermophysics Conference, New Orleans, LA, 1996.

28) Fertig, M., Infed, F., Olawsky, F., Auweter-Kurtz, M. and Adamidis, P.: Recent Improvements of the Parallel-Multiblock URANUS 3D Nonequilibrium Code, Springer-Verlag, Berlin, Heidelberg, Germany, Chap. 31, 2005, pp. 293-310, ISBN 3-540-22943-4.

29) Carlson, A. and Bird, G.: Implementation of a Vibrationally Linked Chemical Reaction Model for DSMC, Tech. Rep. TM 109109, NASA, 1994

30) Moss, J. and Bird, G.: Direct Simulation of Transitional Flow for Hypersonic Re-entry Conditions, AIAA-Paper 84-0223, 1984.

31) Infed, F., Olawsky, F. and Auweter-Kurtz, M.: Stationary Coupling of 3D Hypersonic Nonequilibrium Flows and TPS Structure with URANUS, Journal of Spacecrafts and Rockets, 42 (2005), pp. 9-21.

32) Jahn, G.: Theoretische und experimentelle Untersuchung des Hitzeschutzverhaltens einer Rückkehrkapsel, Ph.D. thesis, Institut für Raumfahrtsysteme, Universität Stuttgart, Germany, 1998, (in German). 